\title{
Cellular and signaling mechanisms supporting cadmium tolerance in salicylic acid treated seedlings
}

\author{
Aïcha Belkadhi*, Wahbi Djebali, Hédia Hédiji, Wided Chaïbi \\ Faculty of Sciences of Tunis, Physiology and Biochemistry of Plant Response to Abiotic Stresses Unit, University of Tunis El Manar, \\ 1060, Tunis El Manar, Tunisia
}

\begin{abstract}
Article history
Received: 5 January 2016

Accepted: 13 February 2016

Published: 18 February 2016
\end{abstract}

(C) Belkadhi et al. (2016)

Editor

K. K. Sabu

\section{Publisher}

Horizon e-Publishing Group

Corresponding Author

Aïcha Belkadhi

\aicha585@yahoo.ca

\begin{abstract}
This review spotlights on recent indications that recognizes potential cellular mechanisms that may be involved in the tolerance of salicylic acid (SA)-treated seedlings to the presence of cadmium (Cd) in their environment. It appears probable that SA stimulates signaling systems implicated in plant defense-related actions against $\mathrm{Cd}$-induced oxidative stress. These include mechanisms that reduce uptake of metals into the cytosol by extracellular chelation through extruded ligands and binding onto cell-wall constituents. Cellular chelation of metals in the cytosol by a range of ligands (peptides, phytochelatins (PCs)), or increased efflux from the cytosol out of the cell or into sequestering compartments are also key mechanisms improving tolerance. Freeradical scavenging capacities through the activity of antioxidant enzymes or production of peptides and PCs add another line of defense against the toxic effect of $\mathrm{Cd}$. The SA signaling events can be attributed to the extracellular SA perception model in which reactions between SA and apoplastic proteins result in acute oxidative burst under Cd stress.
\end{abstract}

\section{Keywords}

Cadmium tolerance; salicylic acid; intracellular chelation; extracellular chelation; signal transduction

Belkadhi, A., Djebali, W., Hédiji, H., and Chaïbi, W. 2016. Cellular and signaling mechanisms supporting cadmium tolerance in salicylic acid treated seedlings. Plant Science Today 3(1): 41-47. http://dx.doi.org/10.14719/pst.2016.3.1.180

\section{Introduction}

Exposure to cadmium (Cd) may occur naturally from metalliferous rocks, or from anthropogenic sources such as industrial pollution inputs, is with high level of acute toxicity for humans and animals (Irfan et al., 2013; 2015). For plants, the degree of metal toxicity depends mainly on the concentration, way and duration of exposure as well as the age, genetics, and nutritional status of exposed seedlings (Djebali et al., 2002). Furthermore, $\mathrm{Cd}$ bioavailability is a function of biotic factors such as mycorrhizal fungi and bacteria (Hall, 2002). In view of this, salicylic acid (SA) is one of the key hormonal factors determining the providence of plants grown on contaminated sites, which is naturally found in plants and shown to be involved in the alleviation of Cd toxicity (Guo et al., 2007, 2013; Li et al., 2014; Belkadhi et al., 2015 $a, b)$.

The local SA signaling actions can be recognized to (i) the extracellular SA action model and/or (ii) intracellular SA perception model (Kawano and Bouteau, 2013). On the other hand, the long distance SA action could be credited to three singular approaches, namely, iii) local increase in SA followed by transportation of SA, (iv) systemic propagation of SA developed movable signals without direct movement of SA, and (v) synergistic 
proliferation of both SA and movable signals through the tissues and phloem. This includes the alternately repeated secondary signal proliferation and synthesis and/or liberation of SA finally contributing to the systemic spread of SA-derived signals.

Previous studies have recapitulated the available data on amelioration of Cd toxicity by exogenous SA applications (Metwally et al., 2003; Choudhury and Panda, 2004; Guo et al., 2007; Li et al., 2014; Belkadhi et al., 2015 a, b; Xu et al., 2015), and this will be as well considered here. In addition, we will focus on the mechanisms involved in improving physiological, cellular, and molecular responses to Cd stress (Atkinson and Urwin, 2012). In fact, various mechanisms potentially involved in $\mathrm{Cd}$ tolerance have been characterized in SA-treated seedlings and can be described as extracellular (binding to the cell-wall) or intracellular (binding to peptides and transport into intracellular compartments) detoxification systems. Extracellular mechanisms are mainly implied in avoidance of $\mathrm{Cd}$ entry, whereas intracellular systems aim to reduce metal burden in the cytosol. Supplementary antioxidative detoxification mechanisms, which allow the alleviation of reactive-oxygen species (ROS) toxicity, indirectly initiated by $\mathrm{Cd}$, may be part of improvement of tolerance mechanisms in SAtreated seedlings.

\section{Role of $\mathrm{SA}$ in $\mathrm{Cd}$ chelation and compartmentalization}

SA is generally considered as an important agent of improving plant resistance to Cd stress probably through its stimulating action of hydrogen peroxide $\left(\mathrm{H}_{2} \mathrm{O}_{2}\right)$ release from cells (Belkadhi et al., 2014). In particular, SA can form chelate compounds with $\mathrm{Cd}$ ions (Perrin, 1958). The specific mechanism that involves $\mathrm{SA}$ in $\mathrm{Cd}$ chelation is not yet explored (Belkadhi et al., 2012). However, Ooto (1975) hypothesized the belief that free o-hydroxyl group on benzoic acid confers metal chelating characteristic to this molecule. A moderate resistance to heavy metals can be realized by selective $\mathrm{Cd}$ exclusion, moderated uptake, or energetic efflux from the roots, especially by mechanisms leading to lower cytoplasmic Cd contents (Hall, 2002). Nevertheless, $\mathrm{Cd}$ tissue contents were unaffected, both at the whole-plant and organ level, in mesophyll cells and vacuoplasts, ruling out the involvement of differential transport of $\mathrm{Cd}$ between plant organs and across the plasma membrane as a physiological reason for the beneficial effect of SA (Metwally et al., 2003).

Members of the ABC transporter family are identified to be implicated in vacuolar sequestration of toxic metals (Kim et al., 2007; Kang et al., 2011). Transcript levels of some Arabidopsis ABC transporters are modified in response to SA (Kang et al., 2011). Such transporters may improve vacuolar sequestration of Cd in the SA-treated seedlings (Metwally et al., 2003). Metwally et al. (2003) also showed that SA content was increased in Cd-stressed seedlings and the possible formation of stable SA-Cd complexes that lowered Cd toxicity after SA pretreatment was expected. Likewise, Cd-SA complex formation in the hydroponics solution is an improbable reason for the favorable effect of SA since the exposure to $\mathrm{Cd}$ started $3 \mathrm{~d}$ after the SA pretreatment in the presoaking experiment and $24 \mathrm{~h}$ afterward in the pretreatment experiment (Belkadhi et al., 2013; 2014). However, complex formation might have played a role in the short-term experiments with leaf slices (Metwally et al., 2003).

\section{SA and complexation of Cd by peptides}

Despite intracellular chelation capacities of tolerant plant species to heavy metals, certain amount of toxic free $\mathrm{Cd}$ ions may be concentrated in the cytosol. Pre-treatment with SA has been shown to improve various mechanisms to cope with the presence of free $\mathrm{Cd}$ ions inside cells. These include molecular and biochemical modifications of root anatomy, the binding of $\mathrm{Cd}$ ions to the cell wall, the transportation of $\mathrm{Cd}$ complexes to the vacuoles for storage, the production of fewer toxic compounds by ways of methylation, and the excretion or secretion of $\mathrm{Cd}$ ions (Djebali et al., 2002; 2005). The most frequent compounds involved in $\mathrm{Cd}$ tolerance are metalbinding peptides and phytochelatins (PCs). The former are cysteine-rich, found mostly in plants, capable of neutralizing toxic $\mathrm{Cd}$ ions and contain glutamic acid in a $\delta$-bond, allowing them to form thiolate bonds with Cd ions (Rao et al., 2006) and thus protect plant cells from damage. On the other hand, PCs are important not only due to their metal-binding capacity, but also because they transport the bound metal ions from the cytoplasm into the vacuoles, where they can be stored as nontoxic form, bound to organic acid ligands (Brunetti et al., 2011; 2015).

One of the earliest studies to report on the protective effect of SA against $\mathrm{Cd}$ exposure of seedlings dealt with the role of PCs in the SAmediated protection against $\mathrm{Cd}$ toxicity. Grown hydroponically in solution containing $0,0.01$, 0.015 , or $0.025 \mathrm{mM} \mathrm{Cd}$, Szalai et al. (2013) reported that presoaking seeds of Zea mays in $0.5 \mathrm{mM} \mathrm{SA}$ before exposure to $\mathrm{Cd}$ reduced the level of heavy metal injury and has an effect on the composition of individual PCs; however this protection was not directly connected with the altered regulation of PCs. Lately, Freeman et al. (2005) have recommended a model for the involvement of SA in the Ni tolerance of Thlaspi hyperaccumulators. Indeed, SA activates Ser-acetyltransferase (EC 2.3.1.30) (Freeman et al., 2004) post-translationally leading to increase in GSH synthesis and activation of GSH reductase (Knörzer et al., 1996) to preserve an improved pool of reduced GSH. Furthermore, 
SA potentially blocks PC synthase activity (Pál et al., 2002), slowing down PC biosynthesis in response to Ni and shielding GSH to operate as an antioxidant (Freeman et al., 2005), thus preventing Ni-induced oxidative stress in Thlaspi hyperaccumulators. In an earlier experiment, it was also proved that the GSH-mediated $\mathrm{Ni}$ tolerance mechanism observed in Nihyperaccumulating Thlaspi species is signaled by the constitutively elevated levels of SA. It was also observed that both biochemical and genetic manipulations that increase SA in Arabidopsis thaliana seedlings imitate the GSH-related phenotypes of the hyperaccumulating Thlaspi, and that these biochemical changes in the nonaccumulator plants are associated with increased GSH-mediated Ni resistance (Freeman et al., 2004). Such results suggested that SA may perhaps be one of the regulators implicated in monitoring certain key biochemical differences between Ni/Zn hyperaccumulators and non-accumulator plant species. Purposely, tolerance of Thlaspi hyperaccumulators to $\mathrm{Cd}$, could be affected by a similar mechanism (Freeman et al., 2005). In any case, the role of SA and GSH to prevent oxidative stress in different plant species showed different levels of $\mathrm{Cd}$ tolerance that still needs to be elucidated.

\section{Influence of $S A$ on transport mechanisms involved in Cd tolerance}

There has been little recent work on the transport of heavy metals in SA-treated seedlings. Apoplastic proteins may be involved in Cd tolerance either by extruding the toxic metal ions from the cytosol out of the cell or by allowing metal sequestration into intracellular compartments (Williams et al., 2000; Hall, 2002). Using radiotracer flux analyses, the significant accumulation of $\mathrm{Cd}$ found in the higher plants, involves the accumulation of $\mathrm{Cd}$-conjugated peptides or Cd-conjugated PCs in the vacuole. This process appears to be mediated by the ATP-binding cassette transporters located at the tonoplast (Ortiz et al., 1995). In fact, ATP-binding cassette (ABC) transporters are known to be involved in vacuolar sequestration of toxic metals (Clemens, 2006). A new $\mathrm{ABC}$ transporter has been identified as a SAinduced gene from Glycine max (Eichhorn et al., 2006). This transporter might facilitate vacuolar sequestration of $\mathrm{Cd}$ in the SA-treated plants. Belkadhi et al. (2015b) assumed that the beneficial effects of exogenous SA pre-treatment can be related to modification of $\mathrm{Cd}$ compartmentalization in vacuoles. As, this molecule can form a complex with $\mathrm{Cd}$ that may provide tolerance to $\mathrm{Cd}$ stress (Moussa and ELGamal, 2010). Otherwise, it is well known that $\mathrm{Cd}$ entry via essential element transporters is the basis process of its uptake by root cells (Hall and Williams, 2003). This absorption and transport of $\mathrm{Cd}$ happens because of the similarity in the electron structure between the heavy metal and essential cations (Cosio et al., 2004).
Additionally, Singh et al. (2015) reported that exogenous application of SA reverted the growth, and oxidative stress caused by arsenic V (As V) and significantly decreased As translocation to the shoots. Then, the level of iron (Fe) in root and shoot was completely interrelated with the transcript level of transporters responsible for the accumulation of Fe, OsNRAMP5, and OsFRDL1, in both plant organs. The authors concluded that these observations might be due to SA-induced down regulation of As transporters from root to shoot. In a previous study OsLsi2, transporter responsible for AsIII transport from root to shoot in rice (Ma et al., 2008), has been found to be down regulated at mRNA level. Since AsIII is the principal intracellular form (Ma et al., 2008; Mishra et al., 2013) and also probably the main As groups translocated to the shoots. Consequently, down regulation of OsLsi2 would negatively affect the As accumulation. Furthermore, Srivastava et al. (2014) explained that down regulation of OsLsi2 was owed to minor As concentration in rice shoots in response to thiourea supplementation with As. OsLsi1 is primarily responsible for AsIII transport to root was not related to As uptake in roots (Singh et al., 2015; Armendariz et al., 2016). Otherwise, SA has been revealed to activate $\mathrm{ABC}$ transporters in Glycine $\max$ (Eichhorn et al., 2006). These transporters are responsible for the vacuolar sequestration of As(III)-PC forms (Song et al., 2010). Therefore, it may be probable that the lesser As transported to the shoot was due to the fact that most of the accumulated As in SA treated rice seedlings were sequestered in root vacuoles as As(III)-PC form. Further, SA pre-treatment has been described to improve PCs synthesis in maize roots (Szalai et al., 2013).

Since SA mediated resistance against $\mathrm{Cd}$ stress has reported in previous studies (Metwally et al., 2003; Shi and Zhu, 2008), some reductions in the level of accumulation were observed (Belkadhi et al., 2010). For this purpose, less concentration of metalloid in shoot may also have an effect on its level in grain which would have great implications with respect to human toxicity through food chain $\mathrm{Cd}$ contamination. Besides, the beneficial effect of SA on Cd bioaccumulation could be attributed to the augmentation of the uptake of cations ( $\mathrm{Ca}, \mathrm{Mg}$ and Fe) in Cd-treated plants (Belkhadi et al., 2010). Within soybean cells, Dean and Mills (2004) reported that SA is converted primarily, in the cytoplasm, to SA 2-O-beta-d-glucose (SAG) and then accumulates, wholly, in the vacuole.

However, the mechanism involved in the vacuolar transport of SAG has not been investigated. By measuring the [(14)C]SAG uptake into tonoplast vesicles isolated from etiolated soybean hypocotyls, the authors characterized the vacuolar transport of SAG. They found that the uptake of SAG was stimulated about 6-fold when Mg-ATP was included in the assay media. In contrast, after adding Mg-ATP, the uptake of SA was only stimulated by 1.25 -fold and was 2.2 -fold 
less than the uptake of SAG providing an indication that the vacuolar uptake of SA was promoted by glucosylation. Based on the characteristics of SAG uptake into soybean tonoplast vesicles, authors concluded that this uptake occurred through an $A B C$ transporter-type mechanism. Nevertheless, this uptake mechanism in vacuoles is not common since the uptake of SAG by Beta vulgaris $\mathrm{L}$. tonoplast vesicles required an $\mathrm{H}\left({ }^{+}\right)$-antiport mechanism (Dean and Mills, 2004).

\section{SA induced oxidative signaling events leading to Cd stress adaptation}

Systemic signals are observed in separate plant tissues and initiate systemic stress resistance through priming or else stimulation of defense reactions (Aranega-Bou et al., 2014). Recently, the knowledge on such long-distance signaling has been documented through the studies on systemic acquired resistance (SAR), systemic acquired acclimation (SAA), and systemic wound response (SWR) (Aranega-Bou et al., 2014). According to previous studies, phloem is the likely path for systemic transmission or movement of signals associated with those three SA-induced responses. Correspondingly to SAR induction following the challenges by abiotic environmental conditions can be the elicitors for SA-centered signaling cascade finally leading to SAA in the challenged plants.

Reactive oxygen species (ROS) are often unavoidably generated by living organisms as byproducts from normal metabolic reactions including respiration and photosynthetic processes, and fatty acid metabolism (Moller, 2001; Baker et al., 2006; Noctor et al., 2012). A widespread property of all forms of ROS is that they can cause oxidative damage to cellular components such as proteins, DNA, and membranes (Moller et al., 2007). The specificity of the biological response of living plant cells to ROS counted on the chemical characteristics of ROS, strength of the signal, sites of production, and growth stages (Del Rio et al., 2002). Consequently, apart from their harmful action, generation of ROS could be potentially advantageous to living cells depending on the environmental conditions (Apel and Hirt, 2004). Induced production of ROS like hydrogen peroxide $\left(\mathrm{H}_{2} \mathrm{O}_{2}\right)$, superoxide anion radicals $\left(\mathrm{O}_{2}{ }^{\circ}\right)$ and hydroxyl radicals (HO'), at the cell walls via the apoplast pathway (Bolwell et al., 2002) recognized as the "oxidative burst", is one of the earliest events detectable during Cd stress (Olmos et al., 2003; Sharma et al., 2012).

Exposures to $\mathrm{Cd}$ stress reportedly increase the intra- and intercellular levels of $\mathrm{H}_{2} \mathrm{O}_{2}$ by modulating the finely highly structured ROS detoxification networks, composed of ROSproducing enzymes, antioxidant enzymes, and biosynthetic pathways for low molecular weight antioxidants, all responsible for maintaining the homeostasis of ROS levels (Bolwell et al., 2002; Del
Ri'o et al., 2002; Kawano et al., 2003; Kotchoni and Gachomo, 2006; Yoshioka et al., 2008). Olmos et al. (2003) demonstrated a rapid generation of $\mathrm{H}_{2} \mathrm{O}_{2}$ by $\mathrm{Cd}^{2+}$-treated plant cells was in cultured tobacco (Nicotiana tabacum L.) BY-2 cells. By using cytochemical methods, they had located the starting point for the generation of $\mathrm{H}_{2} \mathrm{O}_{2}$ at the cell plasma membrane. Exogenous application of diphenyleneiodonium (DPI) and imidazol, both inhibitors of the neutrophil NADPH oxidase, prohibited the production and propagation of $\mathrm{H}_{2} \mathrm{O}_{2}$ induced by $\mathrm{Cd}^{2+}$. These data suggested the involvement of an NADPH oxidase-like enzyme leading to $\mathrm{H}_{2} \mathrm{O}_{2}$ generation through $\mathrm{O}_{2}{ }^{-}$ dismutation by superoxide dismutase enzymes. Then, to investigate the implication of $\mathrm{Ca}^{2+}$ channels in a $\mathrm{Cd}^{2+}$-induced oxidative burst, Olmos and collaborators (2003) had used different inhibitors of $\mathrm{Ca}^{2+}$ channels. Results showed that only $\mathrm{La}^{3+}$ totally inhibited the generation of $\mathrm{H}_{2} \mathrm{O}_{2}$ induced by $\mathrm{Cd}$ ions. Nevertheless, the inhibitors of $\mathrm{Ca}^{2+}$ channels (verapamil and nifedipine) were not effective. Furthermore, based on the results obtained with fluphenazine and N-(-6-aminohexyl)-5-chloro-1- naphthalenesulphonamide (W7) and staurosporine, two types of calmodulin antagonists, an inhibitor of protein kinases, calmodulin or a $\mathrm{Ca}^{2+}$ - dependent protein kinase was also implicated in the signal transduction sequence. However, neomycin, an inhibitor of the phosphoinositide cycle, did not inhibit the formation of $\mathrm{H}_{2} \mathrm{O}_{2}$ induced by $\mathrm{Cd}^{2+}$, signifying essentially an induction of the oxidative burst mediated by calmodulin and/or calmodulindependent proteins.

A series of previous and recent works (Olmos et al., 2003; Sharma et al., 2012) demonstrated for the first time that ROS generation occurs in plants after the exposure to the heavy metal and that the members of ROS possibly function as the chemical signals necessary for induction of hypersensitive response (HR) which is believed to be mediated by endogenous SA (Coll et al., 2011; De Pinto et al., 2012). This allows ROS to serve as signaling molecules in plants in order to regulate the metabolism and the cell signal transduction pathways activated in response to the environmental stress (Gechev et al., 2006; Mittler et al., 2011).

Collected evidences established that hormonal signaling pathways inducing development of SAR are regulated under restricted ROS generation as observed for SA, jasmonic acid (JA), and abscisic acid (ABA) (Kawano, 2003; Mori and Schroeder, 2004; Al-Hakimi, 2007; Gaupels et al., 2011; Maksymiec, 2011; Wen et al., 2011; Kumar et al., 2012; Masood et al., 2012). Similar hormonal regulations mediated by ROS might be involved in the cross talk between abiotic stress signaling pathways (Stroher and Dietz, 2006). Although many constituents of oxidative signaling network pathways have been identified in recent times, the mechanisms for orchestrated control of the 
expanded ROS generation processes at diverse cellular sites through the improvements of ROS feedback control to congregate the physiological necessities such as plant growth and development and adaptation to biotic and abiotic stress are currently actively studied (Coll et al., 2011).

On the other hand, many studies reported that the Cd stress signal might also be transmitted by SA molecules (Rodríguez-Serrano et al., 2006; Yakimova et al., 2006; Krantev et al., 2008; Stroiński et al., 2013). Moreover, accumulation of SA under Cd stress has been noted in pea, maize and Arabidopsis plants (Rodríguez-Serrano et al., 2006; Zawoznik et al., 2007). Additionally, Poovaiah et al. (2013) revealed that expression of genes controlling the defense responses after the accumulation of SA seemed to be regulated by $\mathrm{Ca}^{2+} /$ calmodulin-mediated signaling. In fact, calmodulin is a signal molecule which is crucially involved in the signaling cascades that generate the Cd-induced oxidative burst (Garnier et al., 2006). Besides, as a member of a family of crucial leucine zipper (bZIP) transcription factors for which competitive binding to calmodulin has been shown to be implicated in regulating the levels of expression of different genes (Neuhaus et al., 1994).

Szymanski et al., 1996 showed that TGA3 (a member of the family of transcription factors TAGs) binding to calmodulin, enhances its interaction with the target promoter. Furthermore, TGA3 interrelates with NON-EXPRESSOR OF PATHOGENESIS-RELATED GENES1 (NPR1), one of the most important transcription cofactor involved in SA signal perception and the expression of a large range of genes controlling the defense responses; affording a possible option to regulate the output of defensive reactions (Fu and Dong, 2013). As a final point, it is very interesting to investigate the role of $\mathrm{Ca}^{2+} /$ calmodulin in SAtreated seedlings after the exposure to $\mathrm{Cd}$.

\section{Conclusion}

This review has assessed current researches that discovered potential cellular and signaling mechanisms that may be involved in the tolerance of SA-treated plants to the presence of $\mathrm{Cd}$ in their environment. These include improving mechanisms that decrease uptake into the cytosol by intracellular chelation of metal by means of ligands (peptides, PCs), or efflux from the cytosol into sequestering compartments or binding onto cell-wall components. Specific features such as the efficiency of PC synthesis have also been described. However, most of the molecular mechanisms prolong to be revealed, among which transport mechanisms are of great interest. Moreover, observations with a particular mode of application of SA in plants exposed to $\mathrm{Cd}$ must be generalized with caution. It appears for example that the foremost mechanism implicated in $\mathrm{Cd}$ detoxification in SA-treated plants consists on enhancing its compartmentation within the vacuole as sulphur-rich complexes, whereas $\mathrm{Cd}$ tolerance achievement could be primarily because of a reduced $\mathrm{Cd}$ accumulation within shoot and root cells. Away from these mechanisms, certaintly is the setback of understanding tolerance in SAtreated plants, and this brings in supplementary level of complexity that is beyond the scope of this review. SA cellular signaling mechanisms are likely linked through the import and export of SA by the cells. Since the low-affinity SA efflux carrier required for excretion of highly accumulated SA is reportedly induced by extracellular SA-induced oxidative burst and calcium signaling mechanism.

\section{Competing Interests}

The authors declare that they have no competing interests.

\section{Acknowledgements}

We would like to thank present and previous researchers as well as the reviewers for contributing to the present research topic.

\section{References}

Aranega-Bou, P., M. de la O Leyva, I. Finiti, P. GarcíaAgustín, and C. González-Bosch. 2014. Priming of plant resistance by natural compounds. Hexanoic acid as a model. Frontiers in Plant Science 5: 488. doi: 10.3389/fpls.2014.00488. PMID: PMC4181288.

Armendariz, A.L., M.A. Talano, C. Travaglia, H. Reinoso, A.L. Wevar Oller, and E. Agostini. 2016. Arsenic toxicity in soybean seedlings and their attenuation mechanisms. Plant Physiology and Biochemistry 98: 119-127. doi: 10.1016/j.plaphy.2015.11.021. PMID: 26686284.

Atkinson, N.J., and P.E. Urwin. 2012. The interaction of plant biotic and abiotic stresses: from genes to the field. Journal of Experimental Botany 63: 35233543. doi: 10.1093/jxb/ers100. PMID: 22467407.

Belkadhi, A., H. Hediji, Z. Abbes, W. Djebali, and W. Chaïbi. 2012. Influence of salicylic acid pretreatment on cadmium tolerance and its relationship with non-protein thiol production in flax root. African Journal of Biotechnology 11 : 9788-9796. doi: 10.5897/AJB11.2051.

Belkadhi, A., A. De Haro, P. Soengas, S. Obregón, M.E. Cartea, W. Djebali, and W. Chaïbi. 2013. Salicylic acid improves root antioxidant defense system and total antioxidant capacities of flax subjected to cadmium. OMICS : A Journal of Integrative Biology 17(7): 398-406. doi: 10.1089/omi.2013.0030. PMID: 23758477.

Belkadhi, A., A. De Haro, P. Soengas, S. Obregón, M.E. Cartea, W. Chaïbi, and W. Djebali. 2014. Salicylic acid increases tolerance to oxidative stress induced by hydrogen peroxide accumulation in leaves of cadmium-exposed flax (Linum usitatissimum L.). Journal of Plant Interactions 9(1): 647-654. doi: 10.1080/17429145.2014.890751.

Belkadhi A., A. De Haro, S. Obregon, W. Chaïbi, and W. Djebali. 2015. Positive effects of salicylic acid pretreatment on the composition of flax 
plastidial membrane lipids under cadmium stress. Environmental Science and Pollution Research 22 (2): 1457-1467. doi: 10.1007/s11356014-3475-6. PMID: 25163565.

Belkadhi A., A. De Haro, S. Obregon, W. Chaïbi, and W. Djebali. 2015. Exogenous salicylic acid protects phospholipids against cadmium stress in flax (Linum usitatissimum L.). Ecotoxicology and Environmental Safety 120: 102-109. doi: 10.1016/j.ecoenv.2015.05.028. PMID: 26057076.

Belkhadi, A., H. Hediji, Z. Abbes, I. Nouairi, Z. Barhoumi, M. Zarrouk, W. Chaïbi, and W. Djebali. 2010. Effects of exogenous salicylic acid pre-treatment on cadmium toxicity and leaf lipid content in Linum usitatissimum L. Ecotoxicology and Environmental Safety 73: 1004-1011. doi: 10.1016/j.ecoenv.2010.03.009. PMID: 20399499.

Brunetti, P., L. Zanella, A. Proia, A. De Paolis, G. Falasca, M.M. Altamura, L. Sanita' di Toppi, P. Costantino, and M. Cardarelli. 2011. Cadmium tolerance and phytochelatin content of Arabidopsis seedlings over-expressing the phytochelatin synthase gene AtPCS1. Journal of Experimental Botany 62: 55095519. doi: 10.1093/jxb/err228. PMID: 21841172.

Brunetti, P., L. Zanella, A. De Paolis, D. Di Litta, V. Cecchetti, G. Falasca, M. Barbieri, M.M. Altamura, P. Costantino, M. Cardarelli. 2015. Cadmiuminducible expression of the ABC-type transporter AtABCC3 increases phytochelatin-mediated cadmium tolerance in Arabidopsis. Journal of Experimental Botany 66: 3815-3829. doi: 10.1093/jxb/erv185. PMID: 25900618.

Choudhury, S., and S.K. Panda. 2004. Role of salicylic acid in regulating cadmium induced oxidative stress in Oryza sativa L. roots. Bulgarian Journal of Plant Physiology 30: 95-110.

Clemens, S. 2006. Toxic metal accumulation, responses to exposure and mechanisms of tolerance in plants. Biochimie 88: 1707-1719. doi:10.1016/j.biochi.2006.07.003. PMID: 16914250.

Cosio, C., E. Martinoia, C. Keller. 2004. Hyperaccumulation of cadmium and zinc in Thlaspi caerulescens and Arabidopsis halleri at the leaf cellular level. Plant Physiology 134: 716-725. doi: 10.1104/pp.103.031948. PMID: 14730081.

Djebali, W., W. Chaibi, and M.H. Ghorbel. 2002. Croissance, activité peroxydasique et modifications ultrastructurales induites par le cadmium dans la racine de tomate. Canadian Journal of Botany 80 : 942-953. doi: 10.1055/s-2005837696.

Djebali, W., M. Zarrouk, R. Brouquisse, S. El Kahoui, F. Liman, M.H. Ghorbel, and W. Chaïbi. 2005. Ultrastructure and lipid alterations induced by cadmium in tomato Lycopersicon esculentum chloroplast membranes. Plant Biology 7: 258-368. doi: 10.1055/s-2005-837696. PMID: 16025408.

Eichhorn, H., M. Klinghammer, P. Becht and R. Tenhaken. 2006. Isolation of a novel ABCtransporter gene from soybean induced by salicylic acid. Journal of Experimental Botany 57: 2193-2201. doi:10.1093/jxb/erj179. PMID: 16720608.

Freeman, J. L., M.W. Persans, K. Nieman, C. Albrecht, W. Peer, I.J. Pickering, and D.E. Salt. 2004. Increased glutathione biosynthesis plays a role in nickel tolerance in Thlaspi nickel hyperaccumulators. Plant Cell 16: 2176-2191. doi: 10.1105/tpc.104.023036. PMID: 15269333.

Freeman, J. L., D. Garcia, D. Kim, A. Hopf, and D.E. Salt. 2005. Constitutively elevated salicylic acid signals glutathione-mediated nickel tolerance in Thlaspi nickel hyperaccumulators. Plant Physiology 137: 1082-1091. doi: 10.1104/pp.104.055293. PMID: 15734913.

Guo, B., Y.C. Liang, Y.G. Zhu, and F.J. Zhao. 2007. Role of salicylic acid in alleviating oxidative damage in rice roots (Oryza sativa) subjected to cadmium stress. Environmental Pollution 147: 743-749. doi:10.1016/j.envpol.2006.09.007.

PMID: 17084493.

Hall, J.L. 2002. Cellular mechanisms for heavy metal detoxification and tolerance. Journal of Experimental Botany 53: 1-11. doi: 10.1093/jexbot/53.366.1. PMID: 11741035.

Hall, J.L., and L.E. Williams. 2003. Transition metal transporters in plants. Journal of Experimental Botany 54: 2601-2613. doi: 10.1093/jxb/erg303. PMID:14585824.

Kang, J., J. Park, H. Choi, B. Burla, T. Kretzschmar, Y. Lee, and E. Martinoia. 2011. Plant ABC transporters. Arabidopsis Book 9: e0153 10.1199. doi: 10.1199/tab.0153. PMID: 3268509.

Kawano, T., and F. Bouteau. 2013. Crosstalk between intracellular and extracellular salicylic acid signaling events leading to long-distance spread of signals. Plant Cell Reports 32: 1125-1138. doi: 10.1007/s00299-013-1451-0. PMID: 23689257.

Kim D.Y., L. Bovet, M. Maeshima, E. Martinoia, Y. Lee. 2007. The ABC transporter AtPDR8 is a cadmium extrusion pump conferring heavy metal resistance. Plant Journal 50: 207-218. doi: 10.1111/j.1365-313X.2007.03044.x. PMID: 17355438 .

Knörzer, O. C., B. Lederer, J. Durner, and P. Böger. 1999. Antioxidative defense activation in soybean cells. Physiologia Plantarum 107: 294-302. doi: 10.1034/j.1399-3054.1999.100306.x.

Li, X., L. Ma, N. Bu, Y. Li, and L. Zhang. 2014. Effects of salicylic acid pre-treatment on cadmium and/or UV-B stress in soybean seedlings. Biologia Plantarum 58: 195-199. doi: 10.1007/s10535-0130375-4.

Ma, J. F., N. Yamaji, N. Mitani, X.Y. Xu, Y.H. Su, S.P. McGrath, and F.J. Zhao. 2008. Transporters of arsenite in rice and their role in arsenic accumulation in rice grain. Proceedings of the National Academy of Sciences U.S.A. 105: 9931993510. doi: 10.1073/pnas.0802361105. PMID: 18626020.

Metwally A., I. Finkemeier, M. Georgi, and K.J. Dietz. 2003. Salicylic acid alleviates the cadmium toxicity in barley seedlings. Plant Physiology 132: 272-281. doi: 10.1104/pp.102.018457. PMID: 12746532 .

Mishra S., G. Wellenreuther, J. Mattusch, H.J. Stärk, and H. Küpper. 2013. Speciation and distribution of arsenic in the nonhyperaccumulator macrophyte Ceratophyllum demersum. Plant Physiology 163: 1396-1408. doi: 10.1104/pp.113.224303. PMID: 24058164 . 
Moussa, H.R., and S.M. EL-Gamal. 2010. Effect of salicylic acid pretreatment on cadmium toxicity in wheat. Biologia Plantarum 54: 315-320. doi: 10.1007/s10535-010-0054-7.

Noctor, G., A. Mhamdi, S. Chaouch, Y. Han, J. Neukermans, B. Marquez-Garcia, G. Queval, and C.H. Foyer. 2012. Glutathione in plants: an integrated overview. Plant, Cell and Environment 35: 454-484. doi: 10.1111/j.13653040.2011.02400.x. PMID: 21777251.

Oota, Y. 1975. Short-day flowering of Lamma gibba G3 induced by salicylic acid. Plant Cell Physiology 16: 1131-1135. doi: 10.12692/ijb/5.9.237-243.

Ortiz, D.F., T. Ruscitti, K.F. McCue, and D.W. Ow. 1995. Transport of metal-binding peptides by HMT1, a fission yeast ABC-type vacuolar membrane protein. Journal of Biological Chemistry 270: 47214728. doi: 10.1074/jbc.270.9.4721. PMID: 7876244.

Pál, M., G. Szalai, E. Horváth, T. Janda, and E. Páldi. 2002. Effect of salicylic acid during heavy metal stress. Acta Biologica Szegediensis 46: 119-120. Perrin, D. D. 1958. Stability of Metal Complexes with Salicylic Acid and Related Substances. Nature 182 : 741 - 742. doi: 10.1038/182741a0. PMID: 13590098.

Rao, V.M., M.P. Latha, T.S. Rao, and G.N. Rao. 2006. Mixed ligand complexes of toxic metal ions with L-glutamic acid and L-methionine in urea-water mixtures. Chemical Speciation and Bioavailability 18: 143. doi: 10.1080/09542299.2006.11073749.

Szalai, G., A. Krantev, R. Yordanova, L.P. Popova, and T. Janda. 2013. Influence of salicylic acid on phytochelatin synthesis in Zea mays during $\mathrm{Cd}$ stress. Turkish Journal of Botany 37: 708-714. doi: 10.3906/bot-1210-6.

Singh, A.P., G. Dixit, S. Mishra, S. Dwivedi, M. Tiwari, S. Mallick, V. Pandey, P.K. Trivedi, D. Chakrabarty, R.D. Tripathi. 2015. Salicylic acid modulates arsenic toxicity by reducing its root to shoot translocation in rice (Oryza sativa L.). Frontiers in Plant Science 6 : 340. doi: 10.3389/fpls.2015.00340. PMID: 26042132.

Srivastava, A. K., S. Srivastava, S. Mishra, S.F. D’Souza, and P. Suprasanna. 2014. Identification of redoxregulated components of arsenate (AsV) tolerance through thiourea supplementation in rice. Metallomics 6: 1718-1730. doi: 10.1039/c4mt00039k. PMID: 25008039.

Williams, L.E., J.K. Pittman, and J.L. Hall. 2000. Emerging mechanisms for heavy metal transport in plants. Biochimica et Biophysica Acta 1465: 104126. doi: 10.1016/S0005-2736(00)00133-4. PMID: 10748249.

Xu, L. L., Z.Y. Fan, Y.J. Dong, J. Kong, and X.Y. Bai. 2015. Effects of exogenous salicylic acid and nitric oxide on physiological characteristics of two peanut cultivars under cadmium stress. Biologia Plantarum 59: 171-182. doi: 10.1007/s10535-0140475-9. 